\title{
High-pressure low-temperature phase transitions and structural development in quasi-two- dimensional transition metal oxychlorides
}

\author{
A.M. Schaller ${ }^{1}$, M. Bykov²,3, E. Bykova ${ }^{2}$, K. Glazyrin4, S. van Smaalen ${ }^{1}$ \\ ${ }^{1}$ Laboratory of Crystallography, University of Bayreuth, Bayreuth, Germany, ${ }^{2}$ Carnegie Institution of Washington, Geophysical \\ Laboratory, Washington DC, USA, ${ }^{3}$ Howard University, Washington DC, USA, ${ }^{4}$ Photon Sciences, Deutsches Elektronen-Synchrotron \\ (DESY), Hamburg, Germany \\ achim.schaller@uni-bayreuth.de
}

The strong interest in $\mathrm{MOCl}(\mathrm{M}=\mathrm{Ti}, \mathrm{V}, \mathrm{Cr}, \mathrm{Fe})$ compounds stems from their nonlinear optical properties in the IR band (CrOCl) [1], their use as intercalation compounds for cathode materials (FeOCl) [2], their use as parent structures for van der Waals heterostructures [3] and especially from their low-dimensional magnetic phenomena [4-6].

MOCl-type compounds are isostructural at ambient conditions with the space group Pmmn and consist of double layers of distorted $\mathrm{MO}_{4} \mathrm{Cl}_{2}$ octahedra, which are connected by van der Waals forces. It has been shown that the magnetic behaviour and the dimensionality of MOCl-type compounds is determined by orbital order of the $3 d$ electron of the transition metal [4-6]. For $\mathrm{M}=\mathrm{V}$, $\mathrm{Cr}$, Fe orbital order leads to strong intra- and interchain exchange couplings, which results in quasi-two-dimensional (2D) magnetic systems that exhibit antiferromagnetic (AFM) order at low temperatures [4,5]. The transition to the AFM state is characterized by a magneto-elastic coupling in the form of a monoclinic lattice distortion that lifts the geometric frustration of the magnetic order on the orthorhombic crystal structure as well as by the formation of an incommensurate modulation of the structure [4,5].

Applying hydrostatic pressure to those compounds allows us to continuously adjust the intra- and interchain exchange parameters through the modification of the octahedral geometry and the metal-to-metal distances. This provides a unique opportunity to study the interplay between magnetic order and pressure-induced structural changes in dependence of the electronic configuration of the transition metal within one single structure type. Pressurizing MOCl compounds to approximately $15 \mathrm{GPa}$ leads to a normal-toincommensurate phase transition, characterized by an optimization of the interlayer packing, which is not associated with changes in the electronic or magnetic structure [7]. This gives us, in addition, the possibility to investigate the effect of the high-pressure structural transition on the magnetic order and vice versa.

The high-pressure (HP) low-temperature (LT) single crystal X-ray diffraction experiments, which were conducted at P02.2/PETRA III above and below $\mathrm{T}_{\mathrm{N}, 1 \mathrm{bar}}$ for pressures up to $40 \mathrm{GPa}$ and temperatures down to $6 \mathrm{~K}$, provides an insight into the HP-LT mechanisms of FeOCl: The magneto-elastic coupling is governed by a monoclinic lattice distortion below $\mathrm{T}_{\mathrm{N}, 1 \text { bar, }}$ whereas an interplay between lattice distortion and significant structural changes takes place above $\mathrm{T}_{\mathrm{N}, 1 \mathrm{bar}}$. These changes enhance, from a geometrical perspective, superexchange interactions up to a pressure of $\approx 15 \mathrm{GPa}$ where the structural HP phase transition gets superimposed on the further structural development. We will present the sequence of phase transitions and the structural development of FeOCl in detail and compare it, where applicable, with the quasi-2D compound $\mathrm{CrOCl}$.

With the described approach and an in-depth analysis of structural changes, we aim at disentangling the magneto-structural correlations in the model system of $\mathrm{MOCl}$ as a function of composition, temperature and pressure in order to facilitate the understanding of low-dimensional magnetic systems in general.

[1] M. Wang, J. Zhang, Z. Wang, C. Wang, S. van Smaalen, H. Xiao, X. Chen, C. Du, X. Xu \& Tao, X. (2020). Adv. Optical Mater. 8, 1901446.

[2] T. Yu, X. Zhao, L. Ma \& Shen, X. (2017). Mater. Res. Bull. 96, 485-490.

[3] N. Mounet, M. Gibertini, P. Schwaller, D. Campi, A. Merkys, A. Marrazzo, T. Sohier, I. E. Castelli, A. Cepellotti, G. Pizzi \& Marzari, N. (2018). Nat. Nanotech. 13, 246-252.

[4] J. Angelkort, A. Wölfel, A. Schönleber, S. van Smaalen \& Kremer, R. K. (2009). Phys. Rev. B 80, 144416.

[5] J. Zhang, A. Wölfel, L. Li \& van Smaalen, S. (2012). Phys. Rev. B 86, 134428.

[6] A. Seidel, C. A. Marianetti, F. C. Chou, G. Ceder \& Lee, P. A. (2003). Phys. Rev. B 67, 020405(R).

[7] M. Bykov, E. Bykova, L. Dubrovinsky, M. Hanfland, H.-P. Liermann \& van Smaalen, S. (2015). Sci. Rep. $5,9647$.

\section{Keywords: transition metal oxychloride; high pressure; phase transition; magnetic order;}

Single crystals were grown in the Laboratory of Crystallography, University of Bayreuth, by Kerstin Küspert. We acknowledge DESY (Hamburg, Germany), a member of the Helmholtz Association HGF, for the provision of experimental facilities and travel cost support. This research has been funded by the Deutsche Forschungsgemeinschaft (DFG, German Research Foundation) - 386411512.

Acta Cryst. (2021), A77, C537 